\title{
A STUDY INTO DESIGNING AN AMBIENT WATER BOTTLE THAT SUPPORTS USERS' WATER-INTAKE TRACKING PRACTICES
}

\author{
Coskun, Aykut; \\ Yıldız, Mert; \\ Yılmazer, Hakan; \\ Genç, Hüseyin Uğur \\ Koc University-Arcelik Research Center for Creative Industries
}

\begin{abstract}
Taking on the challenge of motivating users to drink water regularly, we designed a smart water bottle that can track water intake behavior and inform users about this behavior through ambient feedback. We then conducted two studies to explore the bottle's feedback design from the perspective of users and designers. First, we conducted semi-structured interviews with 10 prospective users and found that they would like to receive personalized, precise, gamified and reminding feedback. Second, we conducted a design workshop with 13 professional designers to explore the range of visualizations that can be used to give feedback. Analyzing these visualizations, we identified three reminder types (augmenting, restoring and balancing) and six visualization styles grouped according to three dimensions of ambient displays (representation fidelity, notification level, aesthetic emphasis). In this paper, we first explain our water bottle concept along with existing solutions. Then, we report the results of these studies. Finally, we discuss the potential implications of the results for our own work as well as for designing ambient displays aimed at supporting users' water intake tracking practices.
\end{abstract}

Keywords: water intake, self-tracking, User centred design, Industrial design, Conceptual design

\author{
Contact: \\ Coskun, Aykut \\ Koc University \\ Koc University Arcelik Research Center for Creative Industries \\ Turkey \\ aykutcoskun@ku.edu.tr
}




\section{INTRODUCTION}

Hydration has a vital role in our lives. Inadequate water intake leads to physiological and psychological problems such as headache, fatigue, infections and stress (Jéquier and Constant, 2010; Palma et al., 2015; Ritz and Berrut, 2005). Our bodies exhibit self-response mechanisms, like sensing the thirst (Greenleaf, 1992) or having a dry mouth, yet these only help in the case of deficiency, thus not reliable in keeping a balanced hydration level. Recent studies showed that almost 30 percent of the adults and more than 50 percent of the teenagers are inadequately hydrated in the US (Chang et al., 2016a), and more than 30 percent of adults are dehydrated in UK (Gibson et al., 2012). This situation provides an opportunity for designing interventions to motivate regular water intake. We took up this opportunity to design a new water bottle (GROW) for individuals who would like to monitor and regulate their water intake behaviour. GROW tracks user's water intake by calculating the change in the water level. Once the user drinks water from the bottle, it gives ambient feedback about the user's progress towards his or her daily water intake goal via a tree image that appears on its surface (Figure 1).
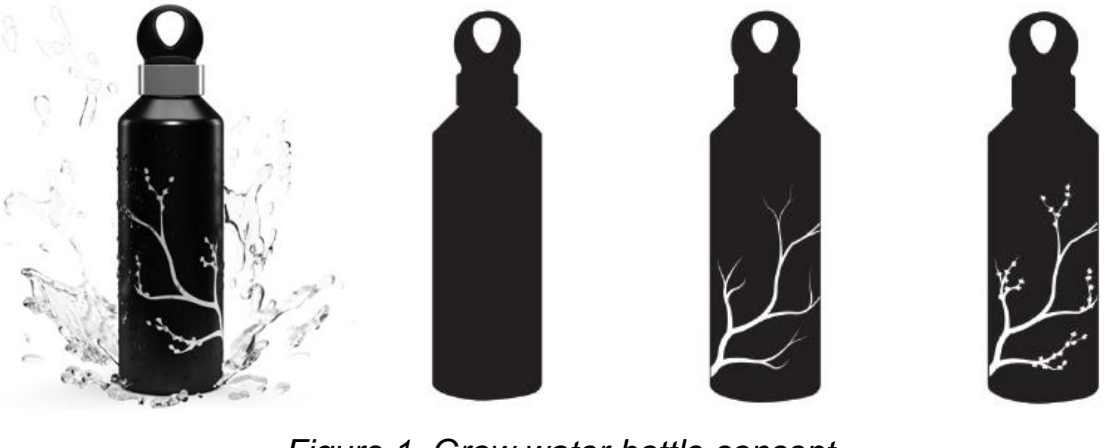

Figure 1. Grow water bottle concept

We then conducted two studies to explore the ambient feedback design from the perspective of users and designers. First, we conducted semi-structured interviews with ten prospective users (office workers who might forget to drink water regularly due to heavy workload and busy schedules) to gather their insights about the concept. As a result, we identified four user expectations: being reminded to drink water, tailoring personalized feedback, providing precise feedback, and integrating gamification into the feedback design. Inspired by the expectation of being reminded, we redesigned our concept. The initial version had two feedback states. When the user does not interact with the bottle, the tree image is dissolved into the bottle surface (idle state). When the user drinks water, the tree image becomes partly or fully visible depending on the user's progress towards his or her goal (progress state). After the first user study, we added a reminder state where the bottle reminds the user to drink water after detecting one-hour inactivity, by showing a partial tree image. Second, we conducted a design workshop with 13 professional designers to explore the design space for the ambient feedback, i.e., the range of visualizations to give feedback about users' water intake through a water bottle surface. We collected 21 visualization designs. We analysed them in terms of how the they reflect the three states (idle, reminder and progress) and identified three reminder types framed as augmenting, restoring, and balancing. We also look at to what extent they cover the feedback design space defined by the dimensions of ambient displays (i.e., information capacity, representation fidelity, notification level and aesthetic emphasis) (Pousman and Stasko, 2006). We found that though the designs represent various visualization styles across the dimensions, majority of them utilizes two types: 1) utilitarian, concrete, and somewhat noticeable, and 2) somewhat aesthetic, concrete, and noticeable. In this paper, after presenting the water bottle concept and the results of these studies, we discuss the implications of these results for our own work and for designing ambient displays aimed at supporting users' water intake tracking practices.

\section{RELATED WORK}

\subsection{Existing solutions to support users in monitoring their water intake}

There has been an interest among researchers in developing solutions that support individuals to monitor their water intake. Mug-Tree (Ko et al., 2007), is a mug with an embedded screen that measures water intake by using a tilt sensor and gives the data through a virtual tree. Watercoaster 
(Lessel et al., 2016) uses a weight sensor to measure the change in the water amount, and gives feedback via a smartphone app. It provides reminders through the use of LEDs and a companion app's notifications. Playful Bottle (Chiu et al., 2009) is a mobile system that uses a camera to determine the water intake and gives notifications to the user via a mobile application. Hydroprompt tracks water intake by a weight sensor (Neves et al., 2016), and aims to motivate users via prompting historical water intake information. It regularly sends notifications to users' computer screen. There are also commercial solutions. For example, Hidrate Spark tracks water intake with its sensor stick extension coming from inside of the cap, and provide reminders through the use of LEDs and companion app notifications which is also used for reflecting on one's daily hydration habits by quantifying the daily intake (Hidrate Inc., 2018). Thermos bottle allows users to manually track their daily water intake with the use of dials or counters embedded on the bottle (Thermos L.L.C, 2018). All of these solutions rely on screen-based interfaces (smart phones) and numbers to give feedback about user's water intake.

\subsection{Using ambient displays to support users' self-tracking practices}

Ambient displays provide users with relevant and timely information without taking too much attention from their primary task. So far, these displays have been used for various purposes, one of which is to track user behaviour and give feedback about this behaviour to support behaviour change. Ambient displays that are designed for this purpose represents a rich design space in terms of targeted behaviours, feedback techniques, and visualization styles. Previous work has explored the ways of tracking and influencing a variety of behaviour including energy and resource conservation (e.g., (Ham and Midden, 2010; Kim et al., 2010a, 2010b; Maan et al., 2011; Nakajima and Lehdonvirta, 2013; Niedderer et al., 2014)) physical activity (e.g., (Jafarinaimi et al., 2005; Mateevitsi et al., 2014; Nakajima and Lehdonvirta, 2013; Rogers et al., 2010)), responsible smartphone use (Choi and Lee, 2016), and better time management (Occhialini et al., 2011). These works also used a variety of techniques to give feedback about user behaviour. These include ambient lighting (e.g., (Ham and Midden, 2010; Maan et al., 2011; Mateevitsi et al., 2014; Occhialini et al., 2011)), actuated displays (Choi and Lee, 2016; Jafarinaimi et al., 2005; Rogers et al., 2010), ambient mirrors (Nakajima and Lehdonvirta, 2013), and sonification (Lockton et al., 2014). In terms of the visualization style of the feedback, while many solutions utilized on abstract representations (e.g., (Ham and Midden, 2010; Jafarinaimi et al., 2005; Maan et al., 2011; Mateevitsi et al., 2014; Occhialini et al., 2011)), others used iconic representations (Ham et al., 2009; Kim et al., 2010a, 2010b), or real-life images or paintings (Nakajima and Lehdonvirta, 2013). Despite these explorations, there has not been an ambient display that tracks and gives feedback about user's water intake behaviour. To connect the work on ambient displays with the work on supporting water intake through design, we designed GROW, a water bottle that uses its own surface as an ambient display to motivate water intake through changes in abstract visuals.

\subsection{GROW: an ambient water bottle concept that tracks and reminds water intake}

GROW tracks water intake via an embedded liquid level sensor, i.e., FDC1004. Once the user drinks, it compares her/his current intake to the intake goal. Based on this comparison, it partially or fully reveals the white silhouette of a cherry tree. After each sip, if the intake amount is not sufficient to reach the user's goal, user only sees the partially revealed branches. If the amount is sufficient, the user also sees the leaves (Figure 1). We intentionally designed GROW's feedback to be unobtrusive, not distracting users from their daily routines but nudging them towards interaction, since it is an essential characteristic of ambient displays (Jafarinaimi, et al., 2005). We used thermochromic paint while implementing the tree image, as its colour slowly changes when exposed to heat.

GROW has some similarities with and differences from existing design solutions (as described in section 2.1.) and ambient displays (as mentioned in section 2.2.). While all of the existing solutions consist of a water container equipped with sensors and a traditional screen-based display to give feedback (Chang et al., 2016b; Hassenzahl, 2008; Hidrate Inc., 2018; Jafarinaimi et al., 2005), GROW uses its own surface as a display to give feedback. Furthermore, as stated above, GROW is one of the first smart bottle concept that gives feedback about users' water intake behaviour with an ambient display. Only example that tracks water intake that uses abstract ambient light to remind to drink water, Hidrate Spark (Hidrate Inc., 2018), uses LED based notifications as reminders. However, GROW distinguishes by giving feedback about user's overall progress, as well as reminding to drink, by using iconic, abstract representations, similar to the work of Kim et al. (Kim et al., 2010a), and Ham et al. (Ham et al., 2009). 


\section{USER INTERVIEWS}

We conducted semi-structured interviews with ten prospective users. We recruited them through e-mails sent to the university's contact directory and our social media accounts. The participants were knowledge workers who work in an office environment. Their ages varied between 26-46 (M=32, SD=5.58). We chose this user group as we believe that due to the rush of work-life, employers tend to forget to drink water regularly. The interviews concentrated on four themes: participants daily routine as it is related to water intake, awareness of the current water intake, insights into our concept and expectations from water bottles aimed at supporting their water intake tracking practices. We prepared a video sketch (Zimmerman, 2005) to illustrate our concept. This video shows a woman working on an open-office desk in front of a computer and drinking water with GROW. After she drinks water and leaves the bottle on the desk, a white cherry tree image appears smoothly. We showed this video right after the participants shared their water intake practices and daily routines. Interviews took approximately 45 minutes. We voice-recorded all the interviews, transcribed these to text, and then analysed them through qualitative coding (Miles,M.B. \& Huberman, 1994) by using interview themes as our analysis scheme. We present the participants' overall reactions to GROW and their expectations of the feedback design.

\subsection{User reactions to the GROW concept}

In general, all the participants were very positive about using GROW. They particularly liked the surprise effect created by the appearance of the tree image after the user drinks from the bottle. Compared to the initial, neutral state of the bottle, the revealing image provided them with rewarding feedback. Half of the participants considered this aspect of GROW as a motivator to drink more water since it provides playful interaction that relates to fulfilling the task (drinking water) and to receive the reward (full tree image). This motivating aspect was consider highly useful because, as participants mentioned, they tend to forget drinking water while they are working, and they think that the existence of GROW on an office table would serve as a reminder to drink water.

\subsection{User expectations}

\subsubsection{Being reminded to drink through notifications}

Participants indicated that a smart bottle should periodically remind them to drink water unless they would forget to drink, even if they have the bottle with them. They attributed this to rush aspect of the work environment they were experiencing. They also wanted to receive feedback for refilling the bottle or refreshing the water inside as they may need to drink more water than the amount inside the container to achieve their daily intake goal. They further wished to customize the frequency of these reminders as they might be annoying sometimes, such as occasional high-intensity work scenarios that they may not want to be disturbed. Another suggestion was about receiving notifications from a mobile app that works as a companion to GROW bottle. They stated that since they are regularly checking their phones, during both work and rest, this would increase the GROW's ability to remind water intake and track users' progress. Note that, based on this expectation, we added a reminder state to our initial concept that allows an intermediary feedback state between neutral and success modes to provide regular feedback.

\subsubsection{Personalized feedback}

This expectation was about personalization of the feedback and personalization of the bottle's visual appearance. Participants stated that making the bottle and the tree image customizable allows the bottle to represent their personalities, e.g., providing colour alternatives or personalizing the image. They emphasized that the required amount of water intake might differ according to individual characteristics and daily activity. They wished that a smart bottle should be able to calculate this amount, unique to each user, by using personal attributes like basal metabolism, height, weight and so on. They also wished to receive personalized and practical health-related info regarding the data collected by the bottle.

\subsubsection{More precise feedback}

While designing GROW, we inspired by the principles proposed in the literature (Jafarinaimi et al., 2005). In these principles, a design intervention which aims to motivate behaviour change should have four characteristics: the feedback should be abstract, non-intrusive, aesthetic and suitable to be viewed in a public setting. GROW's feedback (gradually appearing abstract tree image on the bottle's surface) 
has all of these characteristics. The participants indicated that although this type of feedback allows them to have an overall idea about their progress, receiving more precise information would be more motivating to drink regularly. They said that having two levels would not be enough to know how much water they drank and how much more they need. In this sense, the comments are in line with the idea of integrating a companion app alongside the GROW smart bottle, which might be a good way to maintain the ambient nature of the feedback whilst providing requested precision through another interface.

\subsubsection{Integrating gamification in the feedback}

Even though the participants appreciated the playfulness aspect of revealing tree image, they thought that this aspect could have been further enhanced by using gamification. For instance, they said that level-upping or challenging with friends might create additional motivation for drinking water regularly. The participants further indicated that seeing the same tree image for a long time might reduce its novelty or surprise effect, which might reduce the bottle's impact on sustaining a regular water intake habit.

\section{DESIGN WORKSHOPS}

We conducted a design workshop with 13 professional designers to explore the range of visualizations to give feedback about users' water intake through a water bottle surface. We recruited six graphic designers and seven industrial designers by using authors' designer networks such as mailing lists or alumni platforms, and later via snowballing. These designers had various levels of experience. Six had 0-2 years of experience, 4 had 3-5 years of experience, and 3 had more than five years of experience in the field. We conducted remote workshops, allowing participants to work on their own pace and environment. We prepared a design brief describing the design challenge along with a template that illustrates the tree states of GROW (idle, reminder and progress) with the original tree image. We distributed the brief and the template to the participants and asked them to design one or two visualizations for three states. We gave them two weeks to complete the challenge and received the filled templates via email. We gathered 21 visualizations (Figure 2).

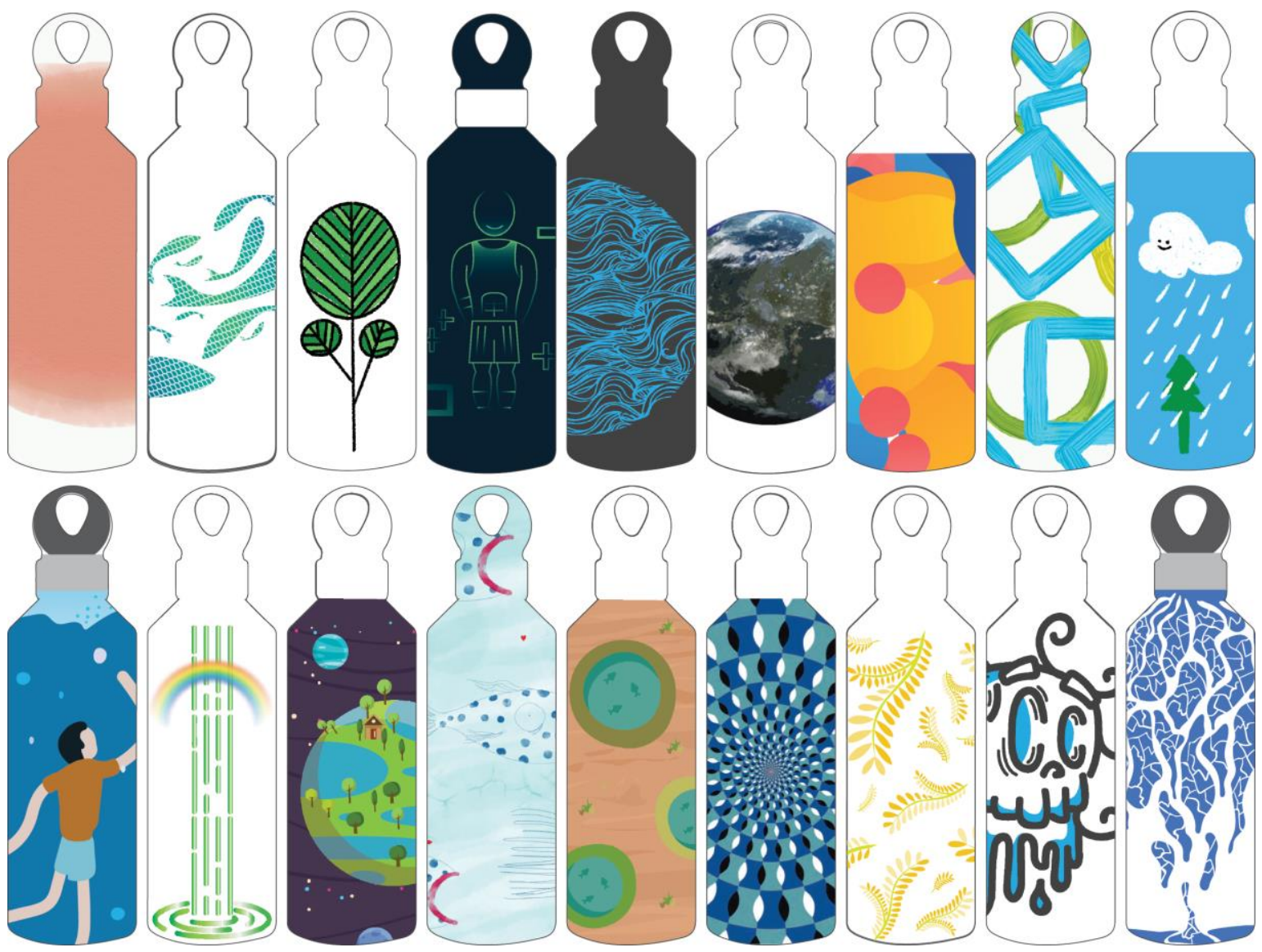

Figure 2. The range of visualizations produced in the remote design workshop 
We analysed these visualizations in terms of how they change to reflect the three states of the bottle. We identified three reminder types which we framed as augmenting (visuals that start with neutral stance and reaches positive), restoring (visuals that move from negative to positive) and balancing (visuals that use positive and negative visuals variably). Besides, we looked at to what extent the visualizations cover the feedback design space defined by the design dimensions of ambient displays. These dimensions are notification level (level of interruption to the user), representational fidelity (how the relevant data is conveyed) and aesthetic emphasis (visual attractiveness) (Pousman and Stasko, 2006). We did not include the information capacity dimension in our analysis since it was kept constant between all concepts; they all had visuals giving feedback using three states of visuals. We created a 7-point semantic differential scale consisting of 3 dimensions to assess the concepts. The dimensions we used for the evaluation were as follows: aesthetic-utilitarian for aesthetic emphasis, abstract-concrete for representational fidelity, and unobtrusive-noticeable for notification level. A group of 7 design researchers working in HCI field, including authors, were given printouts of designs, in addition with keywords and inspirations submitted by designers. Each researcher independently rated concepts according to these adjectives. For example, after looking at a visualization in terms of the notification level dimension, a researcher gives a number between 1 and 7 where 1 refers to "unobtrusive" and 7 refers to "noticeable". Then, individual ratings are gathered to measure homogeneity between ratings given to each visualization. The inter-rater reliability analysis showed that we had a high agreement $(\alpha=.86)$ throughout all dimensions. As a result of this analysis, we created affinity graphs (Figures 6a-6f) with each line representing a visualization and identified six distinct visualization styles amongst them. Majority of the visualizations fell under 2 styles: 1) utilitarian, concrete and somewhat noticeable, and 2) somewhat aesthetic, concrete, and noticeable. We explain these visualization styles along with reminder types by showing examples from the workshops.

\subsection{Reminder types}

\subsubsection{Augmenting reminder}

In this reminder technique, we can see a direct progression from the idle state to the progress state in a linear fashion, and the reminder step is positioned in the middle of this progression. This allows reminder step to be a phase rather than a single visual. This progression starts from a graphically and contextually neutral idle state, as it proceeds the visual becomes more and more enhanced, thus creating a perception of 'development' or 'improvement' from an existing state. Reminder step works as giving a feeling of being unfinished, or as if it is working towards progress state. As in the examples (Figure 3), the idle state can be a planet in space or a neutral visual pattern, which then becomes enhanced, by settlers coming to planet or addition of new graphical units to composition. Thus, the progress state becomes the finalization of this enhanced situation, a civilization on a planet or graphically complete composition. Especially, if the user drinks water after the reminder step, the transformation from the reminder to progress becomes a smooth transition that shows the completion of visuals.
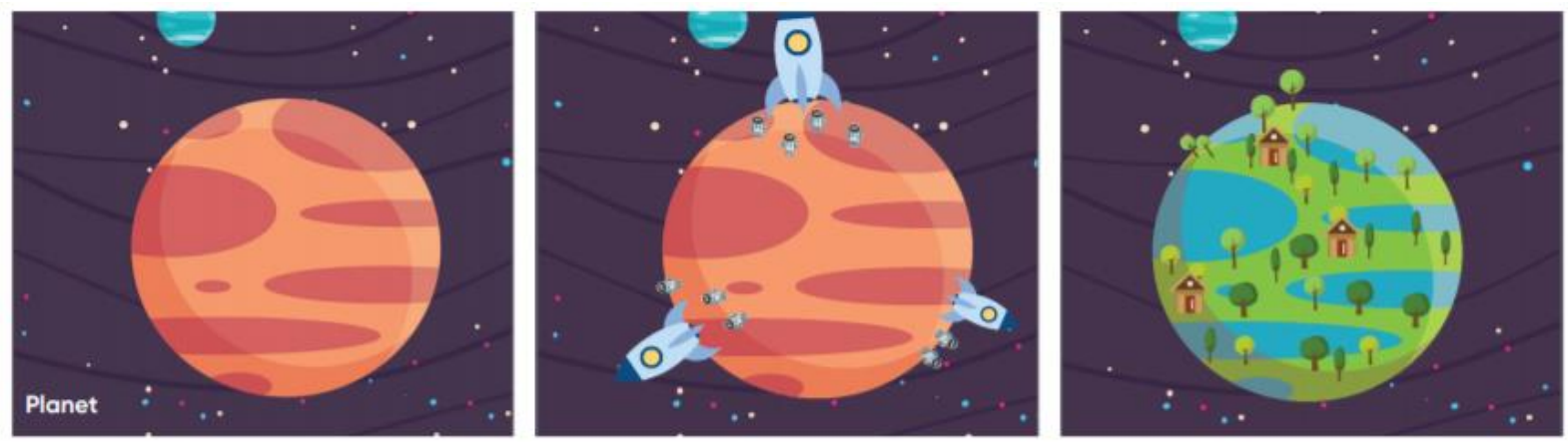

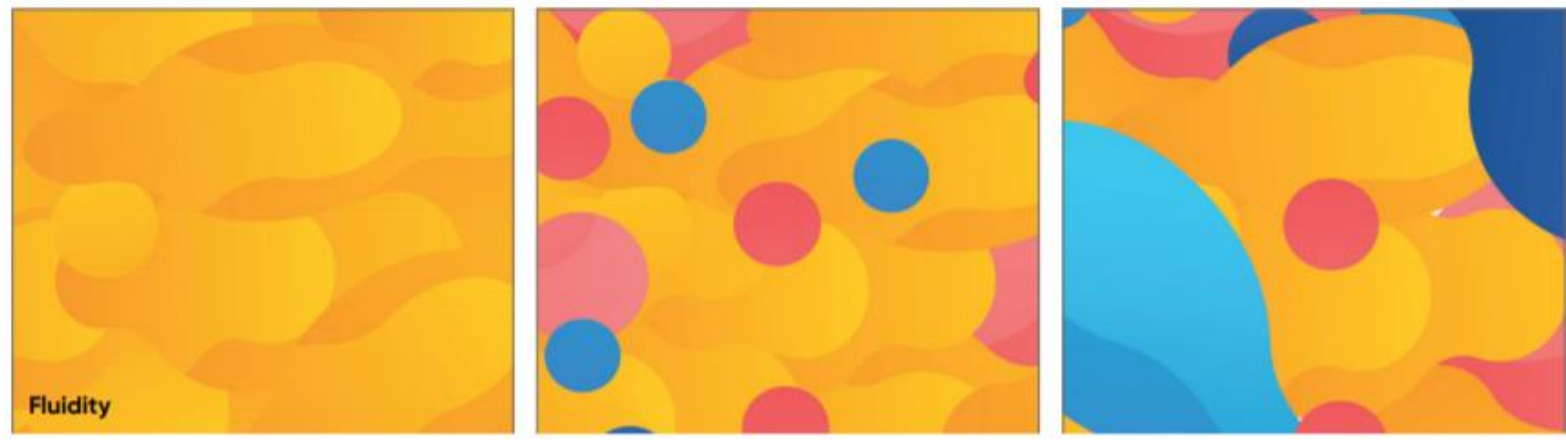

Figure 3. Example visualizations for augmenting reminder

\subsubsection{Restoring reminder}

While the augmenting technique focuses on improving upon a neutral idle state, restoring technique starts up from a negative state, either contextually or graphically. This way, it gives a 'saving' or 'solving a problem' connotation to the user. By building upon it and turning it into a favourable situation, the progress state is achieved. The negative connotation of reminder step gives the feeling of urgency and aims to nudge the user towards solving the problem. Again, reminder state in between this linear progression towards betterment, as user drinks water, the problem visual turns into a more positive outcome. In the workshop examples (Figure 4), this is visualized by using a drowning man or a pattern of dead fish in the idle state. As it moves onto progress state, man gets closer to the surface and more fish becoming alive. Progress states depict a success scenario, the man freed from underwater and all the fish alive.

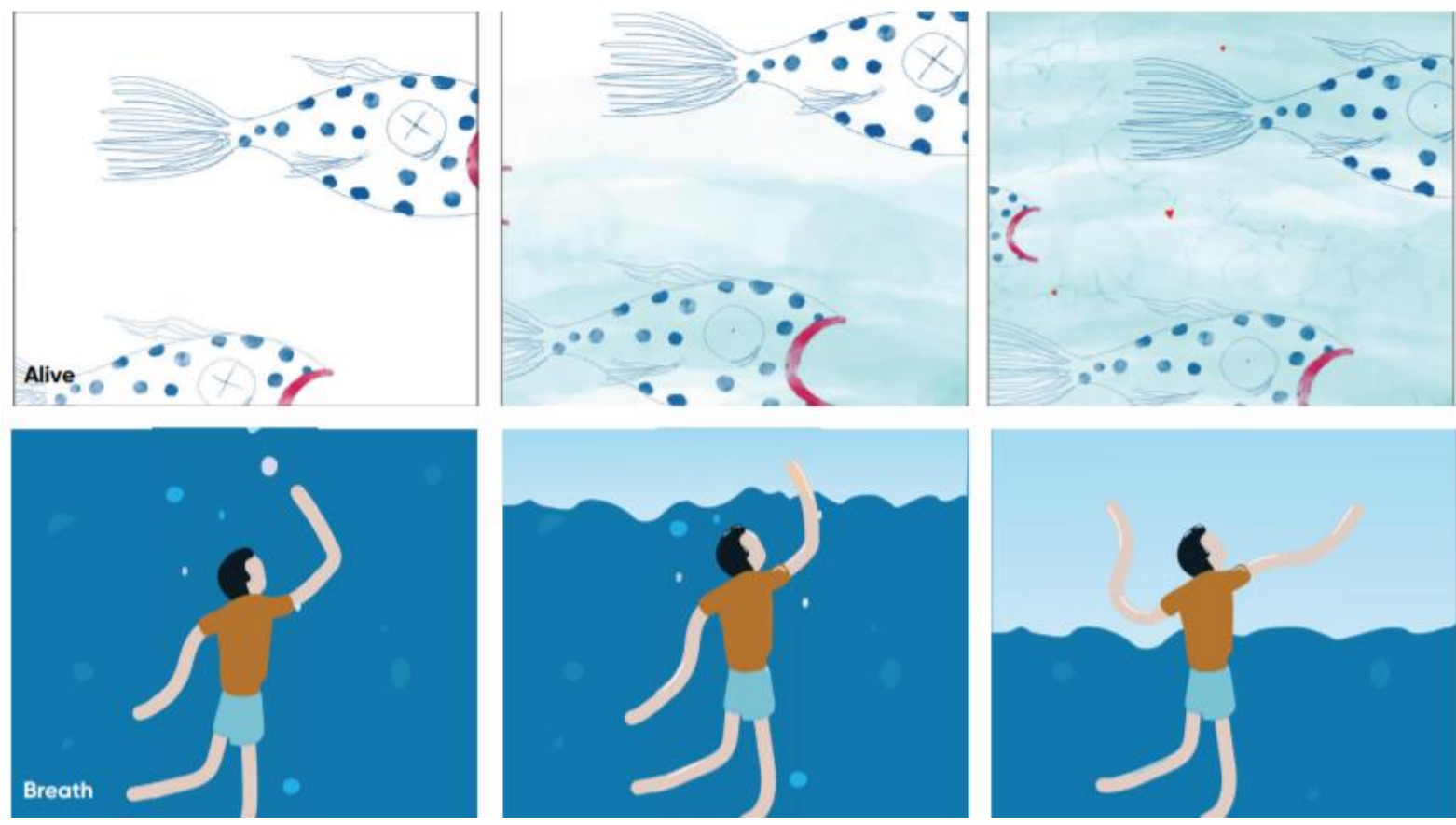

Figure 4. Example visualizations for restoring reminder

\subsubsection{Balancing reminder}

Unlike augmenting and restoring reminders focusing on change from idle to progress, balancing reminder focuses on the change to and from the reminder state specifically. It starts with a neutral state as the idle, but when it comes to the reminder, it shows an undesired situation, which becomes a positive visual in progress. The undesired situation visual in reminder step can metaphorically relate to the effects of dehydration to create a better connection for the user. Aim of these is to motivate the user to balance the negative visuals by taking action to create positive ones. These can be observed in the examples in Figure 5, as idle state shows a regular person or a basic circle, which becomes a 
negative visual for the reminder, using both contextual and graphical elements, like colour. In the progress state visuals become positive both contextually (fitness) and graphically (green).
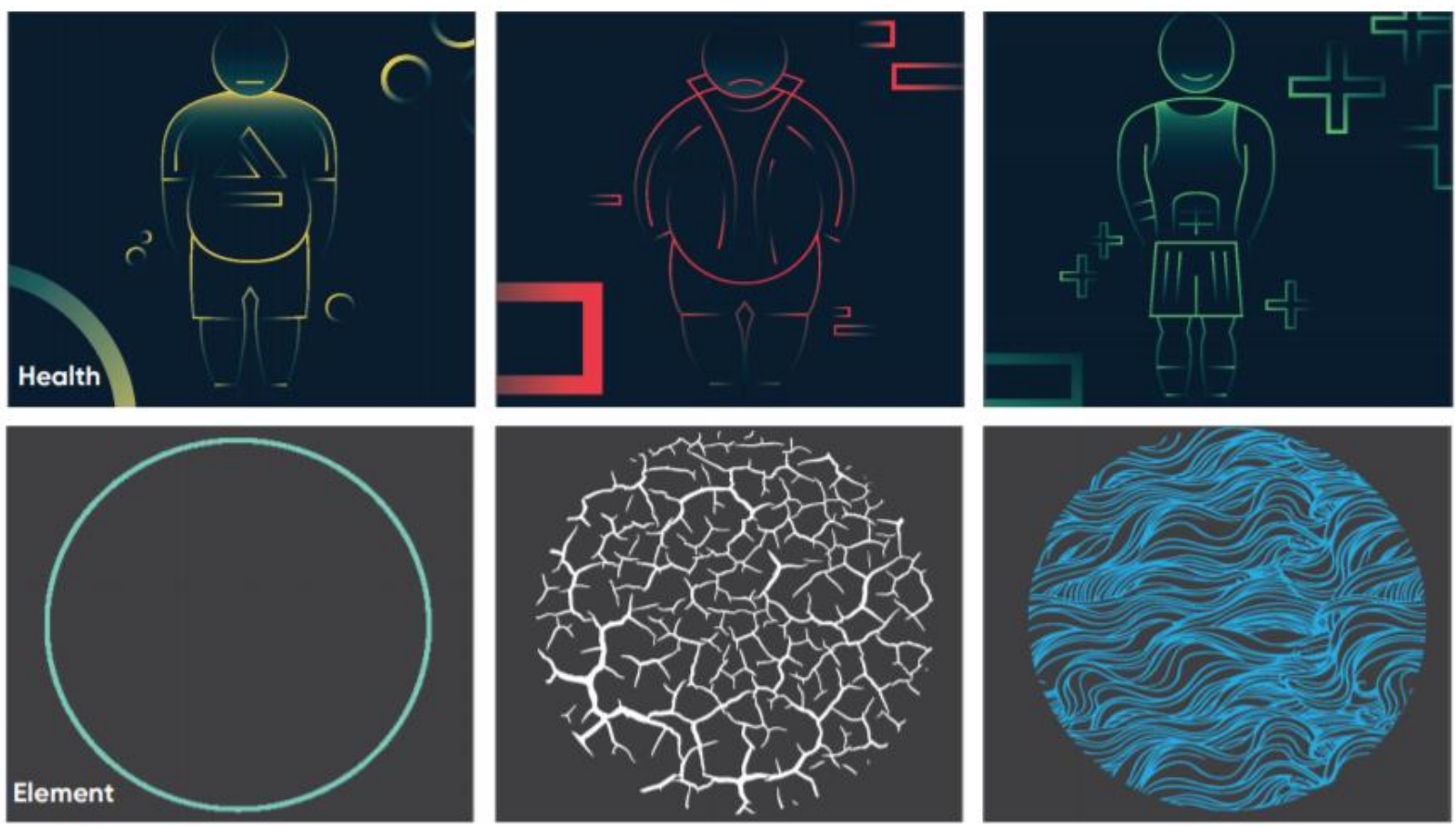

Figure 5. Example visualizations for balancing reminder

\subsection{Visualization styles}

As stated earlier, the visualizations generated in the workshop spread across six styles according to the dimensions of ambient displays. The first style consists of utilitarian, concrete, and somewhat noticeable visuals (n:6) (Figure 6a). The second consist of somewhat aesthetic, concrete and noticeable visuals (n:2) (Figure 6b). Looking at how the styles distributed across visualizations, more than half of the concepts $(n=12)$ followed these two types. The third type consists of aesthetic, abstract and noticeable visuals $(\mathrm{n}: 3)$ (Figure 6c). The fourth consists of somewhat utilitarian, concrete and unobtrusive visuals $(\mathrm{n}: 2)$ (Figure 6d). The fifth consists of aesthetic, abstract and unobtrusive visuals (n:2) (Figure 6e). The last one consists of aesthetic, somewhat abstract and noticeable visuals (n:2) (Figure 6f).

Looking at the path distribution along each dimension, we observed that although there is an evenly distribution in the dimensions of aesthetic emphasis and notification level, the representational fidelity dimension is polarized between abstract and concrete. While 14 concepts and three styles (visualization style 1,4 and 5) included concrete visuals, 5 concepts and two styles (visualization style 3 and 5) included abstract visuals. In other words, it appears that designers had a tendency to use more concrete visuals to give feedback about water intake behaviour.
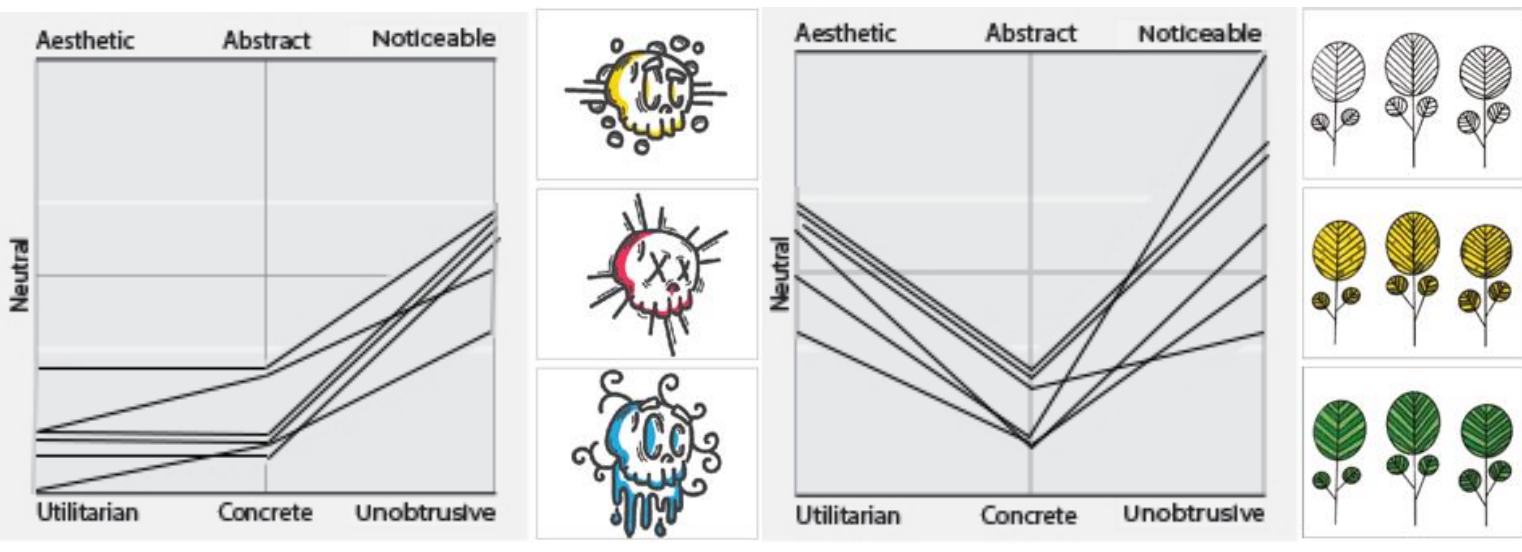

Figure 6a (utilitarian-concrete and somewhat noticeable) and Figure 6b (somewhat aesthetic-concrete and noticeable) 

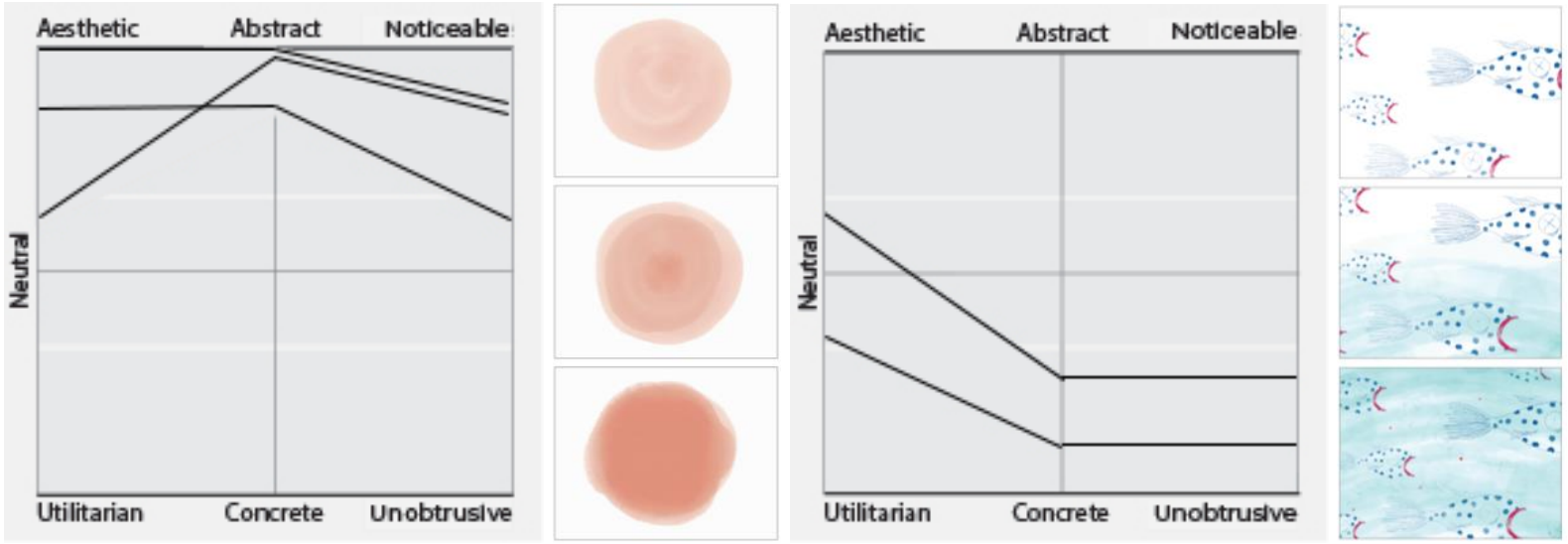

Figure 6c (aesthetic-abstract and noticeable) and Figure 6d (somewhat utilitarian-concrete and unobtrusive)
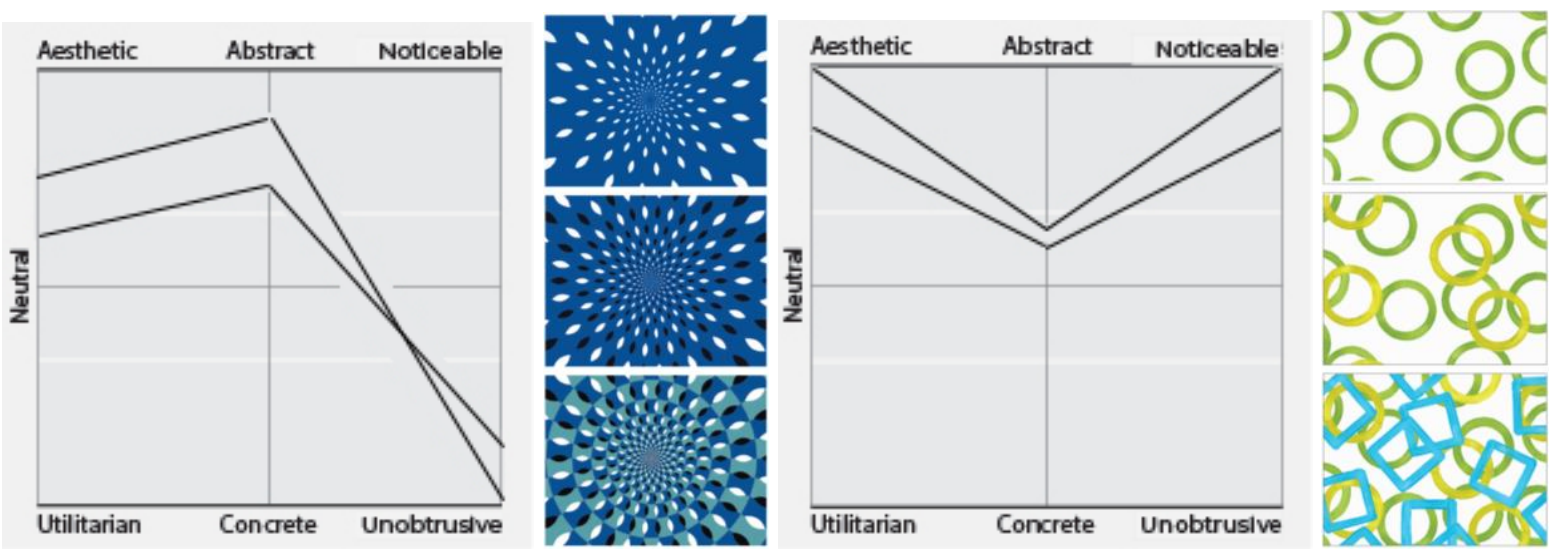

Figure 6e (aesthetic-abstract and unobtrusive) and Figure $6 f$ (aesthetic-somewhat abstract and noticeable)

\section{DISCUSSION}

\subsection{Integration users' expectations into water bottle design}

In this paper, we presented GROW, a water bottle that can track the user's water intake behaviour and inform the user about this behaviour through ambient feedback. We identified four user expectations about this water bottle. Throughout our research process, we integrated one of these expectations, i.e., reminding to drink water, by modifying the feedback states of the bottle. However, we did not integrate other expectations into our bottle design due to various reasons which we explained below. Regarding users' expectations of personalization, previous work indicates that personalization has two significant benefits for designing ambient displays with persuasive intentions. First, personalized feedback can increase the effectiveness of behaviour change strategies (Kreuter and Strecher, 1996). Second, it can contribute to sustained behaviour change via enlengthening the product lifetime (Mugge et al., 2005), as well as strengthening the bond between the user and the product (Chapman, 2012). These issues were also revealed in our interview processes as user insights. However, designing personalized feedback for ambient displays that provide information by using non-conventional means (e.g., using thermochromic paint on the bottle surface as in our concept) would bring some technical challenges. For example, in GROW, revealing the tree visual is achieved by using a heat resistant wire, placed at the backside of the thermochromic paint. Thus, it may be neither technically feasible nor realistic to tailor a unique thermochromic print for every single user, in the context of a product that can be commercially available, depending on their unique needs and desires. Regarding the expectations towards receiving precise feedback, the rationale for giving subtle, ambiguous, and unobtrusive feedback was to avoid distracting users from their primary tasks and revealing their personal information anonymously in a public space. As for the expectations towards utilizing gamification principles into the feedback design, we are aware that these principles have been extensively used in technologies that support health behaviour change (e.g., (Alahäivälä and Oinas-Kukkonen, 2016; Cugelman, 2013)). The expectations of precise feedback 
and gamification could be integrated into the bottle by developing a companion mobile app. In such a scenario, users would see the ambiguous feedback on the bottle but would access detailed and precise information via the mobile app. The app can provide the features that can be used to implement the gamification elements, such as adding users' friends on the map so that they can compete with them or be rewarded with virtual rewards after completing a goal (e.g., seven-day stake). Furthermore, this app could also complement the ambient feedback in situations when the bottle surface is not visible to users (such as in a backpack).

\subsection{Reflections on reminder types and visualization styles in ambient displays}

GROW utilizes augmenting as a reminder type to nudge users to drink water, i.e., no image in the idle state, tree branches in the reminder state and a blossoming tree in the progress stage. In the design workshop, we identified concepts using two other reminder techniques: restoring and balancing. Thus, with this paper, we expanded our initial feedback design space. Future work might explore how different reminder types might influence users water intake behaviour. In addition to that, our analysis of visualizations across ambient display design dimensions revealed interesting insights which indicate directions for future work. Assessing the concepts along the representational fidelity dimension, designers mostly preferred concrete visuals for all the stages, i.e., using a water tap to remind drinking water (Visualization styles 1,2,4). This observation is in line with previous work indicating that choosing concrete visuals over abstract ones in ambient displays may help users understand the relationship between their behaviour and the visual in a better way (Comber et al., 2013). Assessing the concepts along the notification level dimension, we observed that designers commonly used prominent colours or changes in the shapes to make the shifts between stages more noticeable (Visualization styles $1,2,3,6$ ). This is in contrast with the literature indicating that ambient displays are generally unobtrusive (Mankoff et al., 2003) as well as with our initial design. This observation implies that more noticeable reminders may have an affective role in turning water intake behaviour into a habit, rather than unobtrusive reminders. However, this requires future studies to clarify effectiveness over unobtrusive reminders on longitudinal basis for behaviour adoption.

\section{CONCLUSIONS AND FUTURE WORK}

In this paper, we presented a new smart bottle concept aimed at supporting users' self-tracking practices of their water intake behaviour through giving ambient feedback. Our aim in this paper was to explore the potential of this water bottle concept through 1) a user study examining users' reactions towards and expectations from this concept, and 2) a design workshop that led to the exploration of its feedback design space. Although there might be some feasibility issues in terms of implementing this solution into real life (e.g., users may not place the bottle on a flat surface all the time, or they may carry the bottles in a backpack), addressing such issues is beyond the scope of this exploratory study.

We believe that the user nights, reminder types, and visualization styles presented in this paper would inspire designers to envision new products that can support monitoring water intake behaviour and motivating a balanced hydration. GROW was initially designed for individuals working in office environments who might forget to drink water due to heavy workload. Still, it might be used by other user groups like elderly, children and new moms. Thus, we plan to conduct new user studies in the future to tailor the feedback design to the needs of different user groups. Furthermore, assessing whether the bottle would lead to regular water intake is worth exploring from a behaviour change perspective. Since this assessment is beyond the scope of this paper, we plan to address this opportunity by future studies.

\section{REFERENCES}

Alahäivälä, T. and Oinas-Kukkonen, H. (2016), "Understanding persuasion contexts in health gamification: A systematic analysis of gamified health behavior change support systems literature", International Journal of Medical Informatics, available at:https://doi.org/10.1016/j.ijmedinf.2016.02.006.

Chang, T., Ravi, N., Plegue, M.A., Sonneville, K.R. and Davis, M.M. (2016a), "Inadequate hydration, BMI, and obesity among US adults: NHANES 2009-2012”, Annals of Family Medicine, Vol. 14 No. 4, pp. 320-324.

Chang, T., Ravi, N., Plegue, M.A., Sonneville, K.R. and Davis, M.M. (2016b), "Inadequate hydration, BMI, and obesity among US adults: NHANES 2009-2012”, Annals of Family Medicine, available at:https://doi.org/10.1370/afm.1951. 
Chapman, J. (2012), Emotionally Durable Design: Objects, Experiences and Empathy, Emotionally Durable Design: Objects, Experiences and Empathy, available at: https://doi.org/10.4324/9781849771092.

Chiu, M., Chang, S., Chang, Y., Chu, H., Chen, C.C., Hsiao, F. and Ko, J. (2009), "Playful Bottle : a Mobile Social Persuasion System to Motivate Healthy Water Intake", Proceedings of the 11th International Conference on Ubiquitous Computing, pp. 184-194.

Choi, S. and Lee, U. (2016), "LockDoll : Providing Ambient Feedback of Smartphone Usage within Social Interaction”, CHI Extended Abstracts on Human Factors in Computing Systems, pp. 1165-1172.

Comber, R., Thieme, A., Rafiev, A., Taylor, N., Krämer, N. and Olivier, P. (2013), "BinCam: Designing for engagement with Facebook for behavior change", Lecture Notes in Computer Science (Including Subseries Lecture Notes in Artificial Intelligence and Lecture Notes in Bioinformatics), available at:https://doi.org/10.1007/978-3-642-40480-1_7.

Cugelman, B. (2013), "Gamification: What it is and why it matters to digital health behavior change developers", Journal of Medical Internet Research, available at:https://doi.org/10.2196/games.3139.

Gibson, S., Gunn, P. and Maughan, R.J. (2012), "Hydration, water intake and beverage consumption habits among adults”, Nutrition Bulletin, available at:https://doi.org/10.1111/j.1467-3010.2012.01976.x.

Greenleaf, J.E. (1992), "Problem: Thirst, drinking behavior, and involuntary dehydration", Medicine and Science in Sports and Exercise, available at:https://doi.org/10.1249/00005768-199206000-00007.

Ham, J. and Midden, C. (2010), “Ambient persuasive technology needs little cognitive effort: The differential effects of cognitive load on lighting feedback versus factual feedback", Lecture Notes in Computer Science (Including Subseries Lecture Notes in Artificial Intelligence and Lecture Notes in Bioinformatics), available at:https://doi.org/10.1007/978-3-642-13226-1_14.

Ham, J., Midden, C. and Beute, F. (2009), "Can ambient persuasive technology persuade unconsciously? Using subliminal feedback to influence energy consumption ratings of household appliances", ACM International Conference Proceeding Series, available at:https://doi.org/10.1145/1541948.1541988.

Hassenzahl, M. (2008), "User experience (UX)", Proceedings of the 20th International Conference of the Association Francophone d'Interaction Homme-Machine on - IHM '08, p. 11.

Hidrate Inc. (2018), "Hidrate Spark", available at: https://hidratespark.com.

Jafarinaimi, N., Forlizzi, J., Hurst, A. and Zimmerman, J. (2005), "Breakaway: An ambient display designed to change human behavior", Conference on Human Factors in Computing Systems - Proceedings, available at:https://doi.org/10.1145/1056808.1057063.

Jéquier, E. and Constant, F. (2010), "Water as an essential nutrient: The physiological basis of hydration", European Journal of Clinical Nutrition.

Kim, T., Hong, H. and Magerko, B. (2010a), "Design requirements for ambient display that supports sustainable lifestyle", DIS 2010 - Proceedings of the 8th ACM Conference on Designing Interactive Systems, available at:https://doi.org/10.1145/1858171.1858192.

Kim, T., Hong, H. and Magerko, B. (2010b), "Designing for persuasion: Toward ambient eco-visualization for awareness", Lecture Notes in Computer Science (Including Subseries Lecture Notes in Artificial Intelligence and Lecture Notes in Bioinformatics), available at:https://doi.org/10.1007/978-3-642-13226-1_12.

Ko, J.-C., Hung, Y.-P. and Chu, H.-H. (2007), "Mug-Tree: A Playful Mug to Encourage Healthy Habit of Drinking Fluid Regularly", Adjunct Proceedings of the 9th International Conference on Ubiquitous Computing (Ubicomp2007), pp. 220-223.

Kreuter, M.W. and Strecher, V.J. (1996), "Do tailored behavior change messages enhance the effectiveness of health risk appraisal? Results from a randomized trial", Health Education Research, available at:https://doi.org/10.1093/her/11.1.97.

Lessel, P., Altmeyer, M., Kerber, F., Barz, M. and Krüger, A. (2016), "WaterCoaster : A Device to Encourage People in a Playful Fashion to Reach Their Daily Water Intake Level", CHI Extended Abstracts on Human Factors in Computing Systems, pp. 1813-1820.

Lockton, D., Brass, C., Bowden, F. and Gheerawo, R. (2014), "Bird-wattching: exploring sonification of home electricity use with birdsong", No. September.

Maan, S., Merkus, B., Ham, J. and Midden, C. (2011), "Making it not too obvious: The effect of ambient light feedback on space heating energy consumption", Energy Efficiency, available at:https://doi.org/10.1007/s12053-010-9102-6.

Mankoff, J., Dey, A.K., Hsieh, G., Kientz, J., Lederer, S. and Ames, M. (2003), "Heuristic evaluation of ambient displays", Conference on Human Factors in Computing Systems - Proceedings, available at:https://doi.org/10.1145/642640.642642.

Mateevitsi, V., Reda, K., Leigh, J. and Johnson, A. (2014), "The health bar: A persuasive ambient display to improve the office worker's well being”, ACM International Conference Proceeding Series, available at:https://doi.org/10.1145/2582051.2582072.

Miles,M.B. \& Huberman, M. a. (1994), "Qualitative data analysis: An expanded sourcebook (2nd ed.)", Qualitative Data Analysis: An Expanded Sourcebook(2nd Ed.)., Vol. 20 No. 1, pp. 159-160. 
Mugge, R., Schoormans, J.P.L. and Schifferstein, H.N.J. (2005), 'Design strategies to postpone consumers' product replacement: The value of a strong person-product relationship", Design Journal, available at:https://doi.org/10.2752/146069205789331637.

Nakajima, T. and Lehdonvirta, V. (2013), "Designing motivation using persuasive ambient mirrors", Personal and Ubiquitous Computing, available at:https://doi.org/10.1007/s00779-011-0469-y.

Neves, D., Costa, D., Oliveira, M., Jardim, R., Gouveia, R. and Karapanos, E. (2016), "Motivating Healthy Water Intake through Prompting, Historical Information, and Implicit Feedback", pp. 7-10.

Niedderer, K., Mackrill, J., Clune, S., Lockton, D., Ludden, G., Morris, A., Cain, R., et al. (2014), Creating Sustainable Innovation through Design for Behaviour Change: Full Project Report, available at:https://doi.org/10.13140/2.1.4817.4409.

Occhialini, V., Van Essen, H. and Eggen, B. (2011), "Design and evaluation of an ambient display to support time management during meetings", Lecture Notes in Computer Science (Including Subseries Lecture Notes in Artificial Intelligence and Lecture Notes in Bioinformatics), available at:https://doi.org/10.1007/978-3642-23771-3_20.

Palma, L., Marques, L.T., Bujan, J. and Rodrigues, L.M. (2015), "Dietary water affects human skin hydration and biomechanics", Clinical, Cosmetic and Investigational Dermatology, available at:https://doi.org/10.2147/CCID.S86822.

Pousman, Z. and Stasko, J. (2006), "A taxonomy of ambient information systems: Four patterns of design", Proceedings of the Workshop on Advanced Visual Interfaces, available at: https://doi.org/10.1145/1133265.1133277.

Ritz, P. and Berrut, G. (2005), “The Importance of Good Hydration for Day-to-Day Health", Nutrition Reviews, available at:https://doi.org/10.1111/j.1753-4887.2005.tb00155.x.

Rogers, Y., Hazlewood, W.R., Marshall, P., Dalton, N. and Hertrich, S. (2010), “Ambient influence: Can twinkly lights lure and abstract representations trigger behavioral change?", UbiComp'10 - Proceedings of the 2010 ACM Conference on Ubiquitous Computing, available at:https://doi.org/10.1145/1864349.1864372.

Thermos L.L.C. (2018), “Thermos Smart Lid”, available at: https://www.thermos.com/smartlid.

Zimmerman, J. (2005), "Video sketches: Exploring pervasive computing interaction designs", IEEE Pervasive Computing. 